\title{
Daño a la vida de relación como perjuicio autónomo y el daño a la salud
}

\section{The damage to life of relationship, as an autonomous prejudice, and damage to health}

\author{
Claudia Francisca Tarazona Vera ${ }^{* *}$
}

Abogada. Universidad de Pamplona

Cómo citar: Tarazona, C.F. (2016). Daño a la vida de relación como perjuicio autónomo y el daño a la salud. Inciso,18 (2):117-125.

Recibido: 25/06/2015| Revisado: 12/07/2015| Aceptado: 11/12/16

\section{Resumen}

En la investigación se realiza un análisis del daño a la vida de relación a partir del año 2000 y a la salud desde el año 2011, de la jurisprudencia del Consejo de Estado, a fin de establecer la autonomía de cada tesis evidenciando con ello la importancia que tienen en la indemnización del daño inmaterial. De igual forma, se evidencia la falta de justificación que tiene el contencioso al momento de expulsar del ordenamiento jurídico la primera tipología para darle paso al tema del daño a la salud frente a la responsabilidad extracontractual de Estado.

Palabras clave: Daño a la vida de relación, daño inmaterial, perjuicio, Estado, indemnización.

\begin{abstract}
This research analyzes the damage to life of relationship since 2000, and to health since 2011 of the Council of State case law, in order to establish autonomy of each thesis highlighting their importance in indemnifying the non-material damage. Likewise, it is seen the lack of justification by the contentious, at the time of expelling the first type from the legal system, to approach the matter of damage to health, in front of non-contractual liability of the State.
\end{abstract}

Keywords: Damage to life of relation, non-material damage, prejudice, State, compensation. 


\section{Introducción}

El consejo de estado en el año 2000 incorporó la tesis del daño denominado "daño a la vida de relación", indemnizando con ella las secuelas ocasionadas al sujeto en relación con su mundo existencia y cotidiano, cuando se configurase el daño antijurídico y la imputación fáctica y jurídica realizada a la administración pública. Esta tesis se mantuvo prácticamente hasta el 14 de septiembre del año 2011, fecha en donde la alta corporación adoptó la nueva tesis del "daño a la salud", donde quedaban incorporadas todos los tipos de daños inmateriales.

La problemática se presenta al expulsar mediante el daño a la salud, la tesis del daño a la vida en relación, desconociendo su autonomía establecida por el mismo consejo de estado y la corte suprema de justicia. Por tanto, es válida la pregunta ¿Con la aparición del daño a la salud, pierde autonomía la tesis del daño a la vida de relación? Esta es la pregunta que orienta la investigación. Si la respuesta llegase a ser afirmativa, el daño a la salud encuentra toda su justificación, de lo contrario la eliminación del daño a la vida de relación no tendría ninguna justificación.

Se pretende enfrentar dialógicamente dos tesis que propenden por la reparación del daño inmaterial cuando la administración pública es declarada responsable administrativamente por su conducta antijurídica en relación con el administrado.

Iniciar tal tarea se traduce en dejar de manifiesto la autonomía del uno en relación con el otro, es decir, del daño a la vida de relación frente a la nueva tesis del daño a la salud, a fin de establecer si la anulación o expulsión del daño a la vida de relación por parte del consejo de estado con la nueva figura se justifica, o por el contrario, merecía seguir siendo parte de los medios para indemnizar a la víctima de un daño.

Puede concluirse, que el esfuerzo académico resuena en favor de la relación más débil del derecho administrativo, el ciudadano, que contando con medios idóneo para ser indemnizados, se le puedan estar desconociendo derechos fundamentales.

\section{Daño a la vida de relación}

Para estudiar cualquier temática y antes de generar inquietudes e hipótesis se debe establecer algunos tipos de precedentes históricos que marquen los inicios y desarrollos de lo que hoy en día es conocido como "perjuicios fisiológicos o daño a la vida en relación”.

En la aclaración de voto del doctor Ramiro Saavedra becerra expediente $\mathrm{N}^{\circ} 31500$ con radicado 25000 232600020020040901 se hizo claridad en cuento al tratamiento como sinónimos de daño a la vida en relación y perjuicios por alteraciones a las condiciones de existencia pues en los años noventa el Consejo de Estado reconoció una sección adicional a la antigua división de perjuicios patrimoniales y morales, y fue reconocido como perjuicios fisiológicos, que en sí son aquellos que se refieren a la perturbación del pleno goce de la existencia por el hecho de haber sufrido una lesión que afectara el desarrollo de actividades recreativas, culturales, deportivas, el deseo sexual y la capacidad para la realización del mismo (Contencioso de Antioquia, 1992).

Es de recibo, afirmar que el 6 de mayo del año 1993 el Honorable Consejo de Estado en su Sección Tercera Expediente 7428 habló por primera vez del concepto de perjuicios fisiológicos. Con esto ciertamente se aceptaba la existencia de una nueva especie y diferente a las consagradas como perjuicios materiales y morales en la legislación nacional. En el caso de referencia la Sala reconoció perjuicios fisiólogicos pretendidos en una demanda como sinónimo del préjudice d'agrémen - pérdida de agrado, placer $o$ amenidad en el idioma francés -, o a la vida de relación por parte del Consejo de Estado, aun después de esto admitió haber recibido la compensación que representan los perjuicios morales y materiales, la víctima:

seguirá estando muy lejos de la situación privilegiada en que se encontraba antes del hecho dañino, pues no podrá seguir disfrutando de los placeres de la vida. Esto nos indica que el daño moral subjetivo y fisiológico son diferentes (...) la indemnización por perjuicios morales subjetivos repara la satisfacción síquica o el daño físico de la víctima; en cambio, la indemnización del perjuicio fisiológico repara la supresión de las actividades vitales' (Consejo de Estado, 1993). 
Este concepto de daño a la vida de relación se entiende como la privación de los disfrutes y de las satisfacciones que la víctima podría esperar en la vida de no haber ocurrido el accidente, por lo general la doctrina a manera histórica lo ubica desde una perspectiva social, por lo que consideraba que no solo se trataba de la pérdida de la posibilidad de realizar determinada actividad bien sea artística o deportiva etc., y en primer lugar la imposibilidad de llevar una vida en relación ocasionado como resultado del daño corporal realizado a la víctima más la suma de las afectaciones sentimentales.

En Colombia, el llamado daño a la vida en relación fue incluido en la jurisprudencia nacional principalmente en los casos relacionados con la disminución funcional del aparato de locomoción y de los órganos genito-urinarios. El Consejo de Estado en sentencia del 19 de julio del año 2000 dijo que el daño podría ser sufrido por la víctima directamente o también por las personas que normalmente lo rodean, ya sea por motivos de parentesco, amistad o matrimonio, que en ciencia cierta sería no se tratan de perjuicios directos sino indirectos por el tipo de relación con la persona que ha sufrido el daño.

Es evidente que el daño a la vida en relación es un factor que se exterioriza en el sentido en que se refleja en el entorno de las personas mientras el moral se desarrolla de una manera más interna, pero existe una clara relación entre ambos ya que uno configura al otro porque si existe afectación moral alterara la vida de relación del común de las personas.

Para el Consejo de Estado el daño extra patrimonial denominado en sus fallos daño a la vida de relación se refiere a una definición más entendible por lo que se dice que resulta muy inadecuada la sinonimia de perjuicios fisiológicos. En Colombia este perjuicio del cual se hace referencia no alude, solamente a la imposibilidad de gozar placeres de la vida tal cual como hablábamos anteriormente con el controvertido préjudice d'agrement- pérdida de agrado, placer o amenidad en el idioma francés -.

El concepto de daño a la vida en relación, "no consiste en la lesión en sí misma, sino en las consecuencias que, en razón de ella se producen en la vida de relación de quien la sufre" (Consejo de Estado, 2000), evidentemente para el Consejo el daño de la vida en relación es aquel que se encamina a las consecuencias generadas por las lesiones cometidas, cualquiera sea la naturaleza de esa lesión y lo que produce sobre determinada persona haciendo la salvedad de que este perjuicio ocasionado será de una naturaleza diferente de los fisiológicos y de los morales.

Por otra parte, pero sin alejarse mucho la Corte Suprema de Justicia, en reiteradas ocasiones se ha pronunciado sobre el derecho que tienen las personas que haya sufrido un episodio dañoso, a poder reclamar el perjuicio de daño a la vida en relación. "[...]Y el tercero, es el denominada daño a la vida de relación, que se traduce en afectaciones que inciden en forma negativa sobre su vida exterior, concretamente, alrededor de su "... actividad social no patrimonial [...]" " (Corte Suprema de Justicia, 2008).

Es así, como la Corte concluye que el daño a la vida en relación es un derecho inherente a la persona, y que este mismo debería ser reconocido por el ordenamiento, y en este mismo pronunciamiento la Corte hizo una diferenciación entre el daño moral al decir que,

a diferencia del daño moral, que corresponde a la órbita subjetiva, íntima o interna del individuo, el daño a la vida de relación constituye una afectación a la esfera exterior de la persona, que puede verse alterada, en mayor o menor grado, a causa de una lesión infligida a los bienes de la personalidad o a otro tipo de intereses jurídicos, en desmedro de lo que la Corte en su momento denominó "actividad social no patrimonial (Corte Suprema de Justicia, 2008)

Los precedentes históricos son otorgados tanto como por el Concejo de Estado como por la Corte Suprema de Justicia quienes se han encargado de generar un historial amplio sobre el tema de este capítulo y estos mismo son quienes se han encargado de caracterizar el daño a la vida en relación de la siguiente manera:

a.) Es un perjuicio de naturaleza inmaterial o extra patrimonial.

b.) Se refleja en la esfera externa del individuo, aspecto que lo distingue del daño moral.

c.) Tiene múltiples manifestaciones en el entorno personal, social y familiar del afectado. 
d.) Puede originarse de lesiones de tipo físico, y también de otros bienes intangibles de la personalidad o derechos fundamentales.

e.) Puede ser sufrido tanto por la víctima como por terceros como sus familiares o amigos.

f.) Su reconocimiento patrimonial busca aminorar los efectos negativos del daño.

g.) Es un daño autónomo, que se refleja en la vida social de la persona, lo cual no excluye la posibilidad de que sean reconocidos otro tipo de perjuicios.

Hay que decir que, a raíz de la - Sentencia del 19 de julio del 2000 Expediente 11842 - se marcó una evolución histórica respecto al daño a la vida en relación determinando que la Sala asimiló lo que era conocido hasta ese momento como perjuicios fisiológicos en la figura del daño a la vida en relación, la Sala aclaró que el reconocimiento de este perjuicio 'no debe limitarse a los casos de lesiones corporales que proceden alteraciones a nivel orgánico, sino que debe extenderse a todas aquellas situaciones que alteran la vida de relación de las personas: tampoco debe limitarse su reconocimiento a la víctima, toda vez que el mismo puede ser sufrido además por las personas cercanas a ésta, como su cónyuge y sus hijos; ni debe restringirse a la imposibilidad de gozar de los placeres de la vida, pues puede referirse además al esfuerzo excesivo de realizar actividades rutinarias; ni se trata sólo de la afectación sufrida por la persona en su relación con las demás, sino también con las cosas del mundo.

Pero al año siguiente en providencia del 6 de septiembre del año 2001 expediente 13 232-15646, se determinaba una tasa que marcaría el valor de las condenas por los perjuicios morales fijados en moneda legal corriente, y se realizaba la salvedad de que la suma equivalente a 100 salarios mínimos legales mensuales para los eventos en que tales perjuicios cobraran su mayor intensidad, pero sí sería posible determinar en costos monetarios los daños ocasionados a nuestra vida en relación.

\section{El daño a la salud}

En Jurisprudencia del Consejo de Estado - 14 de septiembre de 2011 - trajo el tema a colación e hizo las siguientes apreciaciones de manera categórica con el ánimo de establecer la consistencia del daño a la vida de relación. La Corporación lo definió como "la pérdida de placer en la realización de una actividad o alteración grave que produce el daño en las relaciones del sujeto con su entorno" (Consejo de Estado, 2011).

En este pronunciamiento sobre el tema -daño a la vida de relación - el Consejo de Estado ayudado por la doctrina, la jurisprudencia local y extranjera delinea tal concepto para decir que se constituye por una lesión corporal que trae consecuencias nefastas para el individuo, pues altera su mundo exterior, le desagrada su existencia, lo reduce, lo cosifica y por tanto se hace necesario una reparación integral y no parcial como se pretende en el estado actual de las cosas.

El 14 de septiembre del año 2011, el Consejo de Estado Sección Tercero, en expediente 19031 adoptó y expuso la tesis del "Daño a la Salud" con el fin de sistematizar el tema de la indemnización por daños inmateriales que se veía disperso y daba oportunidad para múltiples indemnizaciones por el mismo hecho generador de los perjuicios. De otra parte es evidente que este pronunciamiento tiene como finalidad la unificación jurisprudencial del tema del daño inmaterial por su importancia jurídica, económica y social en los términos de los artículos 130, 270 y 271 de la Ley 1437 de 2011 (Solano, 2011), pues un tema tan multívoco desde la doctrina, la normatividad y tan costoso para el Estado, con gran impacto social especialmente en las víctimas a la hora de ser compensados "integralmente" por un hecho dañoso desplegado por la Administración Pública, ameritaba una postura como de hecho la ha asumido la Corporación en tal pronunciamiento.

La cuestión es problemática y contraria en muchos aspectos. En términos generales el ponente del proyecto, Dr. Enrique Botero Gil, sintetiza en el concepto de "Daño a la Salud" la indemnización por daños inmateriales que antes se determinaba en las tesis llamadas "alteraciones a las condiciones de existencia" y daño grave a la vida de relación" que había subsumido a aquella. Al respecto se pregona que sólo es indemnizable el "Daño a la Salud" y es probado única y exclusivamente mediante la incapacidad médico legal. Sin embargo, en la sentencia se abre la 
puerta para que se indemnicen otros daños diferentes al "Daño a la Salud" pero que no se sabe cómo se pueden probar, pues la sentencia es categórica en materia de tarifa legal. Es una contradicción de difícil asimilación, pues si lo que se quería era sistematizar la cuestión, en este sentido se hizo fue lo contrario, se dio paso al caos y a la apertura de otras posibles indemnizaciones que atacan el deseo de racionalizar los recursos económicos del Estado. Se da paso a lo conocido como el enriquecimiento sin causa que en el pronunciamiento jurisprudencial pasa de inadvertido y que es punto de controversia en el Salvamento de Voto.

Es aquí donde discrepa la Consejera Dra. Ruth Stella Correa Palacio en el Salvamente de Voto que le hace a la sentencia de fecha 14 de septiembre de 2011, expediente 19031, al decir en términos generales que se propende por un retroceso de la jurisprudencia sobre el tema, pues ya había claridad al respecto en tesis prexistentes que ampliamente han sido mencionadas en este trabajo. También es aceptable la ausencia de claridad y uniformidad a la hora de establecer los criterios bases para la indemnización de forma igualitaria y equitativa, pues con la prueba que se establece varía la liquidación al momento de indemnizar y desplaza el valor de la persona humana como medida del resarcimiento por la lesión a la integridad psicofísica y no una tabla que para ello se imponga. De otro punto la sentencia confunde el "daño evento" con el "daño consecuencia" generando un enriquecimiento injustificado, pues se debe indemnizar, según el pensar de la consejera, las consecuencias que genera el daño y no el daño en sí mismo, pues al pretender el consejero ponente que se pueda indemnizar por otros daños diferentes al "Daño a la "Salud" con otros medios probatorios que la misma sentencia niega al establecer la tarifa probatoria pero al mismo tiempo establece que única y exclusivamente el juez puede reconocer por daños inmateriales el concepto de "Daño a la Salud" se está configurando tal enriquecimiento. En este aspecto la cuestión es de difícil comprensión, pues no se sabe que es lo que se quiere con dicha tesis. Por tanto, se justifica la discrepancia en cuanto al enriquecimiento sin causa, pues si se quería sistematizar el daño inmaterial, se debió acoger una tesis que indemnizara las consecuencias que genera el hecho dañoso y no dar pie al caos y la confusión como lo hace tal jurisprudencia, que en últimas no cumple su cometido.

Una vez expuestos algunos antecedentes críticos de la sentencia del 14 de septiembre del año 2011 como núcleo esencial de este trabajo, se hace necesario establecer de forma cercana el razonamiento del Concejo de Estado en los siguientes términos para ver cómo se estructura la categoría del "Daño a la Salud" y mirar cuál es su consistencia.

En esta oportunidad el Consejo de Estado afirmó que el "Daño a la Salud" se denomina como aquel que resulta de una lesión corporal, es un daño biológico que tiene repercusiones en otras áreas del individuo de talante externo, como son la esfera social, sexual, familiar, en fin todo aquello que sea consecuencia de dicha lesión siempre y cuando se demuestre dentro del proceso

En cuanto a lo comprendido en esta categoría ha dicho el Concejo de Estado que... "sería comprensivo de otros daños como el estético, el sexual, el psicológico, entre otros, sin que existiera la necesidad de ampliar en demasía la gama o haz de daños indemnizables, con lo que se conseguiría una sistematización del daño no patrimonial.” (Consejo de Estado, 2011)

Los componentes de la indemnización - Objetivo y Subjetivo - el "...el daño a la salud se repara con base en dos componentes: i) uno objetivo determinado con base en el porcentaje de invalidez decretado y ii) uno subjetivo, que permitirá incrementar en una determinada proporción el primer valor, de conformidad con las consecuencias particulares y específicas de cada persona lesionada. Así las cosas, el daño a la salud permite estructurar un criterio de resarcimiento fundamentado en bases de igualdad y objetividad, de tal forma que se satisfaga la máxima "a igual daño, igual indemnización"”. (Consejo de Estado, 2011).

La sistematización de los daños inmateriales en la tesis del "Daño a la Salud" a pesar de pregonarse su autonomía, generalidad, abstracción y universalidad en el sistema de daños fundada en los principios de dignidad humana y e igualdad material, no es del toda unívoca y certera, pues deja abierta la puerta para que ha discrecionalidad del Consejo de Estado, en este 
caso, se puedan reconocer otros perjuicios, siempre y cuando estén acreditados como consecuencia de la lesión corporal. De una manera muy sutil lo que hace la Corporación es abrir la puerta para que se configuren más tipologías que lleven a un caos en materia de daños inmateriales.

\section{Reiteración de la filosofía del daño a la salud}

En sentencia del 28 de marzo de $2012^{1}$, el Consejo de Estado reitera la filosofía del denominado "daño a la salud", expuesto en el año anterior, afirmando que éste consiste en el perjuicio proveniente de una afectación psicofísica, estableciendo en una sola categoría los distintos tipos de perjuicios como el físico, psicológico, sexual, entre otros, "de tal forma que siempre que el daño consista en una lesión a la salud, será procedente determinar el grado de afectación del derecho constitucional y fundamental (artículo 49 C.P.) para determinar una indemnización por ese aspecto, sin que sea procedente el reconocimiento de otro tipo de daños". Al respecto la Corporación asevera que:

Se reconoce de este modo una valoración del daño a la persona estructurado sobre la idea del daño corporal, sin tener en cuenta categorías abiertas que distorsionen el modelo de reparación integral. Es decir, cuando la víctima sufra un daño a la integridad psicofísica sólo podrá reclamar los daños materiales que se generen de esa situación y que estén probados, los perjuicios morales de conformidad con los parámetros jurisprudenciales de la Sala y, por último, el daño a la salud por la afectación de este derecho constitucional. Lo anterior, refuerza aún más la necesidad de readoptar la noción de daño a la salud, fisiológico o biológico, como lo hace ahora la Sala, pero con su contenido y alcance primigenio, esto es, referido a la afectación o limitación a la integridad psicofísica de la persona, como quiera que al haberlo subsumido en unas categorías o denominaciones que sirven para identificar perjuicios autónomos y que han sido reconocidos en diferentes latitudes, como por ejemplo la alteración a las condiciones de existencia (v.gr. Francia), se modificó su propósito que era delimitar un daño común (lesión a la integridad corporal) que pudiera ser

1. Consejo de Estado. Sección Tercera. Consejero ponente: Enrique Gil Botero Bogotá 28de marzo de 2012. tasado, en mayor o menor medida, a partir de parámetros objetivos y equitativos, con apego irrestricto a los principios constitucionales de dignidad humana e igualdad ${ }^{2}$.

En otros términos, un daño a la salud desplaza por completo a las demás categorías de daño inmaterial como lo son la alteración grave a las condiciones de existencia - antes denominado daño a la vida de relación - precisamente porque cuando la lesión antijurídica tiene su génesis en una afectación negativa del estado de salud, los únicos perjuicios inmateriales que hay lugar a reconocer son el daño moral y el daño a la salud.

Con todo, se puede ver el propósito de la jurisprudencia citada, consistente en establecer la claridad y la pertinencia del daño a la salud, como la única categoría susceptible de ser indemnizable, por el Estado cuando se configure el daño antijurídico.

Es así, como, "el daño a la salud gana claridad, exactitud y equidad donde los precisados perjuicios la pierden, puesto que siempre está referido a la afectación de la integridad psicofísica del sujeto, y está encaminado a cubrir no sólo la modificación de la unidad corporal sino las consecuencias que las mismas generan, razón por la que, sería comprensivo de otros daños como el estético, el sexual, el psicológico, entre otros, sin que existiera la necesidad de ampliar en demasía la gama o haz de daños indemnizables, con lo que se conseguiría una sistematización del daño no patrimonial ${ }^{3 "}$.

Ahora bien, desde lo que en este apartado se ha denominado, la reiteración de la filosofía del daño a la salud, es evidente el pensamiento del Consejo de Estado, en lo relacionado con sintetizar o sistematizar el tema del daño o perjuicio que, en términos económicos, le cueste menos al Estado Colombiano. En Colombia el sistema indemnizatorio está limitado y no puede dar lugar a que se abra una multiplicidad

2. "El daño subjetivo o daño a la persona es aquél cuyos efectos recaen en el ser humano, considerado en sí mismo, en cuanto sujeto de derecho, desde la concepción hasta el final de la vida. Por la complejidad del ser humano, los daños pueden efectuar alguna o algunas de sus múltiples manifestaciones o "maneras de ser"”. FERNÁNDEZ SESSAREGO, Carlos "El daño a la persona", Revista de la Facultad de Derecho de la Pontificia Universidad Católica del Perú, Lima, pág. 71 y s.s.

3. Fruto del trabajo jurisprudencial en Italia, se llegó a establecer dentro de este orden que el concepto daño biológico agrupa entre otros: el daño a la vida de relación, el daño estético, el daño a la esfera sexual y el daño a la incapacidad laboral genérica, todo lo cual ha sido recogido y reconocido por la Corte Suprema de Casación de ese país. Ver entre otras, las sentencias: No. 2761 de 1990, 1341 de 1991, 11133 de 1990, 9170 de 1994, y 7262 de 1991. 
de categorías resarcitorias que afecten la estructura del derecho de daños y la estabilidad presupuestal que soporta un efectivo sistema de responsabilidad patrimonial del Estado, motivo por el que, se itera, cuando el daño se origine en una lesión psíquica o física de la persona el único perjuicio inmaterial, diferente al moral que será viable reconocer por parte del operador judicial será el denominado "daño a la salud o fisiológico", sin que sea posible admitir otras categorías de perjuicios en este tipo de supuestos $\mathrm{y}$, mucho menos, la alteración a las condiciones de existencia, categoría que bajo la égida del daño a la salud pierde relevancia, concreción y pertinencia para indemnizar este tipo de afectaciones.

En conclusión, y, aludiendo a lo pregonado por el Consejo de Estado, en materia de tipología del daño o del perjuicio,

[...] el concepto de salud comprende diversas esferas de la persona, razón por la que no sólo está circunscrito a la interna, sino que comprende aspectos físicos y psíquicos, por lo que su evaluación será mucho más sencilla puesto que ante lesiones iguales corresponderá una indemnización idéntica ${ }^{4}$. Por lo tanto, no es posible desagregar o subdividir el daño a la salud o perjuicio fisiológico en diversas expresiones corporales o relacionales (v.gr. daño estético, daño sexual, daño relacional familiar, daño relacional social), pues este tipo o clase de perjuicio es posible tasarlo o evaluarlo, de forma más o menos objetiva, con base en el porcentaje de invalidez decretado por el médico legista. "De allí que no sea procedente indemnizar de forma individual cada afectación corporal o social que se deriva del daño a la salud, como lo hizo el tribunal de primera instancia, sino que el daño a la salud se repara con base en dos componentes: i) uno objetivo determinado con base en el porcentaje de invalidez decretado y ii) uno subjetivo, que permitirá incrementar en una determinada proporción el primer valor, de conformidad con las consecuencias particulares y específicas de cada persona lesionada.

"Así las cosas, el daño a la salud permite estructurar un criterio de resarcimiento fundamentado en bases de igualdad $y$ objetividad, de tal forma que se satisfaga la máxima "a igual daño, igual indemnización".

4."Este estado de cosas no sólo deja la sensación de desorden, sino que también crea desigualdades entre víctimas, cuyos intereses aparecen, en igual medida, dignos de protección; así pues, el problema de fondo es el de establecer los límites, que deben ser "límites razonables", determinados sí, en términos jurídicos.” CORTES, Edgar Ob. Cit. Pág. 57.

5. "En el histórico fallo 184 de 1986 la Corte Constitucional italiana afirmó

\section{Autonomía del daño a la vida de relación}

De lo analizado en los anteriores apartada, se determina que las dos categorías de daño tienen autonomía propia, pues se les predica en primer momento su antijuridicidad, certeza y aspecto personal, no permitiendo con ello que uno de estas tipologías subsuma a la otra y por ende deba desaparecer y con ello la indemnización a la víctima.

$Y$ es que el daño antijurídico para ser resarcible, requiere que esté totalmente configurado, por tal motivo, se torna imprescindible que se acrediten los siguientes aspectos relacionados con la lesión o detrimento cuya reparación se reclama:

a.) debe ser antijurídico, esto es, que la persona no tenga el deber jurídico de soportarlo;

b.) que sea cierto, es decir, que se pueda apreciar material y jurídicamente - que no se limite a una mera conjetura -, y que suponga una lesión a un derecho, bien o interés legítimo que se encuentre protegido el ordenamiento jurídico, y

c.) que sea personal, es decir, que sea padecido por quien lo solicita, en tanto se cuente con la legitimación en la causa para reclamar el interés que se debate en el proceso, bien a través de un derecho que le es propio o uno que le deviene por la vía hereditaria.

La antijuridicidad del daño va encaminada a que no solo se constate la materialidad y certidumbre de una lesión a un bien o interés amparado por la ley, sino que, precisamente, se determine que la vulneración o afectación de ese derecho o interés contravenga el ordenamiento jurídico, en tanto no exista el deber jurídico de tolerarlo.

Sólo habrá daño antijurídico cuando se verifique una modificación o alteración negativa fáctica o material respecto de un derecho, bien o interés legítimo que es personal y cierto frente a la persona que lo reclama,

que el criterio de liquidación que debe adoptarse para el resarcimiento del daño biológico "debe, de un lado, responder a una uniformidad pecuniaria de base (el mismo tipo de lesión no puede valorarse de manera diferente para cada sujeto) y, de otro, debe ser suficientemente elástico y flexible para adecuar la liquidación del caso concreto a la incidencia efectiva de la lesión sobre las actividades de la vida cotidiana, por medio de las cuales se manifiesta concretamente la eficiencia sicofísica del sujeto perjudicado." ROZO Sordini, Paolo "El daño biológico", Ed. Universidad Externado de Colombia, Bogotá, pág. 209 y 210. 
y que desde el punto de vista formal es antijurídico, es decir no está en la obligación de soportar porque la normativa no le impone esa carga.

Como se aprecia, el daño antijurídico es el principal elemento sobre el cual se estructura la responsabilidad patrimonial de la administración pública, a la luz del artículo 90 de la Carta Política, entidad jurídica que requiere para su configuración de dos ingredientes: i) uno material o sustancial, que representa el núcleo interior y que consiste en el hecho o fenómeno físico o material (v.gr. la desaparición de una persona, la muerte, la lesión, etc.) y ii) otro formal que proviene de la norma jurídica, en nuestro caso de la disposición constitucional mencionada ${ }^{6}$.

El daño a la vida de relación y el daño a la salud comparten esto aspectos, con la diferencia que el primero tiende a ser más amplio o si se quiere decir integral al momento de establecer la indemnización, pues se mira la sujeto en toda su relación con el exterior o entono cultural, a contrario sensu del daño corporal que se queda a mitad de camino, pues solo le interesa la indemnización del daño corporal más no las repercusiones o consecuencias de tal daño.

Frente a este apartado se tiene presente que Fernández Arbeláez (2014) e un estudio científico sobre el tema ha hecho un estudio sobre el tema y ha indicado que el daño a la salud es inútil e cuanto a la reparación integral de la víctima, pues tiene presente al momento de la indemnización una igualdad formal más no material de las consecuencias del mismo, sin tener en cuenta la dignidad del sujeto

Además de lo anterior, el autor critica el hecho de que ante el nuevo estado de cosas, se confunde las categorías de daño y perjuicio, cuestión que desconoce la reparación integral de la víctima.

Así las cosas, se determina que una cosa es el daño a la persona y otra las consecuencias del mismo, lo cual deben ser indemnizados y que tales cosas se enmarcan dentro del daño a la vida de relación y daño a la salud, si se quisiera cumplir con el cometido de una reparación que propenda por la integralidad indemnizatoria y no se limite la misma con la pretensión de sistematizar la teoría del daño inmaterial diferente a la moral, en la categoría de daño a la salud.

6. Cf. DE CUPIS, Adriano "El Daño", Ed. Bosch, Barcelona, 2a edición, 1970, pág. 82 .

\section{Conclusiones}

El Consejo de Estado sistematizó la tipología de daños inmateriales en la tesis del "daño a la salud" con el argumento de que ésta contenía las demás tesis, sin percatarse de que con ello se propende por una cosificación y materialización de la persona, lo corporal.

El alto órgano de cierre abandona la tesis del daño a la vida de relación, siendo esta universal puesto que comporta una naturaleza inmaterial o extrapatrimonial, refleja en la esfera externa del individuo, aspecto que lo distingue del daño moral, tiene múltiples manifestaciones en el entorno personal, social y familiar del afectado, puede originarse de lesiones de tipo físico, y también de otros bienes intangibles de la personalidad o derechos fundamentales, puede ser sufrido tanto por la víctima como por terceros como sus familiares o amigos, su reconocimiento patrimonial busca aminorar los efectos negativos del daño y es un daño autónomo, que se refleja en la vida social de la persona, lo cual no excluye la posibilidad de que sean reconocidos otro tipo de perjuicios.

De otra parte, el daño a la salud, si bien tiene autonomía no contiene las categorías del daño conocido como daño a la vida de relación. Las categorías del daño a la salud son escasas y reduccionistas, y puede decirse que son propias de la persona. Aquí radica la confusión conceptual de tal institución que se traduce en desconocimiento de derechos fundamentales como, por ejemplo, la reparación integral.

Las categorías o tesis comportan la antijuricidad que se requiere para ser resarcibles, sin embargo, es de anotar que el daño a la salud propende por economizarle dinero al estado, desconociendo derechos fundamentales de la persona en el estado social de derecho.

\section{Referencias bibliográficas}

Bustamante, Á. (2003). La responsabilidad Extracontractual de Estado. Bogotá: Leyer.

Consejo de Estado, 11842. Sección Tercera 19 de julio de 2000. 
Consejo de Estado, 2000300090700, 2003.

Consejo de Estado, 38. 222 y 19.031. Sección Tercera 14 de Septiembre de 2011.

Consejo de Estado, 7428 Sección Tercera 6 de Mayo de 1993.

Consejo de Estado. Sección Tercera. Consejero ponente: ENRIQUE GIL BOTERO Bogotá 28de marzo de 2012.

Contencioso de Antioquia. Tribunal Contencioso Administrativo de Antioquia 3 de julio de 192.

Corte Suprema de Justicia. Sala de Casación Civil. M.P. Cesar Julio Valencia Copete, 13 de mayo de 2008.

Cortés. E. (2009). Responsabilidad civil y daños a la persona. El daño a la salud en la experiencia italiana, ¿un modelo para América Latina? 2009. Bogotá. Universidad Externado de Colombia.

Fernández, S. C. (1993). Daño y protección a la persona humana. Buenos Aires. Ediciones la roca.

Gil, E. (2011). Responsabilidad Extracontractual del Estado. Quinta Edición. Temis. Bogotá.

Gil, E.(2006). Temas de responsabilidad extracontractual del Estado. Bogotá: Comlibros.Marín, H. (2007). Discrecionalidad Administrativa. Bogotá: Externado de Colombia.

Henao, J C. (1998). El Daño. Análisis comparativo de la responsabilidad extracontractual del Estado en derecho colombiano y francés. Bogotá.

Fernández I, (2014). Daño a la salud: perjuicio inmaterial inútil e inconveniente para reparar integralmente el daño. Inciso vol (16) p 139-151.
Koteich. M. (2012). La reparación del daño como mecanismo de tutela de la persona. Del daño a la salud a los nuevos daños extrapatrimoniales. Bogotá. Universidad Externado de Colombia.

M"causland, M.C. (2008) Tipología y reparación del daño no patrimonial. Situación en Iberoamérica y en la jurisprudencia de la Corte Interamericana de Derechos Humanos. 2008. Bogotá. Universidad Externado de Colombia.

Rozo, P. (2002). El Daño Biológico. Bogotá. Universidad Externado de Colombia. 